\title{
Complex Partial Fraction Decompositions of Rational Functions
}

\section{Ali Özyapici ${ }^{*}$ and Cornel S. Pintea ${ }^{2}$}

${ }^{1}$ Girne American University, Department of Engineering, Kyrenia, Mersin 10, Turkey

${ }^{2}$ Babe,s-Bolyai University, Faculty of Mathematics and Computer Sciences, 400084 M. Kogalniceanu 1, Cluj-Napoca, Romania

\begin{abstract}
This work is aimed to obtain the complex partial fraction decompositions of rational functions. We express the coefficients of complex partial fraction decomposition of arbitrary rational functions in terms of the coefficients of their real partial fraction decomposition. This type of decompositions is then used to generalize the high order derivatives of such rational functions. Moreover, different applications are selected to demonstrate the applicability of introduced algorithms.
\end{abstract}

Keywords: Partial fraction; Complex partial fraction; Decomposition; Rational functions

\section{Introduction}

Rational functions are widely used in many branches of mathematics suchasnumericalanalysisi.ePadeApproximations, mathematicalanalysis as well as mathematical modelling and they appear in mathematical representations of many problems in science and engineering [1-7]. Unfortunately, working with rational functions except polynomials is generally not very easy. From the point of view, some methods can be used to facilitate the computations. One of the famous and simplest methods is partial fraction decomposition method (PFD) for suitable applications: Consider a polinomial function $Q(x)$ with real coefficients and recall that there exist some integers $k_{1}, \ldots, k_{p}, l_{1}, \ldots, l_{q} \geq 1$ such that $Q(x)=\left(x-x_{1}\right)^{k_{1}} \ldots\left(x-x_{p}\right)^{k_{p}}\left(x^{2}-2 \operatorname{Re}\left(z_{1}\right) x+\left|z_{1}\right|^{2}\right)^{l_{1}} \ldots\left(x^{2}-2 \operatorname{Re}\left(z_{q}\right) x+\left|z_{4}\right|^{2}\right)^{l_{4}}$

and observe that $Q(x)$ can be equally represented as

$\left.\left(x-x_{1}\right)^{k_{1}} \ldots\left(x-x_{p}\right)^{k_{p}}\left(x-z_{1}\right)^{l_{1}}\left(x-\bar{z}_{1}\right)^{l_{1}}\right)^{l_{1}} \ldots\left(x-z_{4}\right)^{l_{4}}\left(x-\bar{z}_{4}\right)^{l_{4}}$,

where $x_{1}, \ldots, x_{p} \in \mathbb{R}$ are pairwise different and $z_{1}, \ldots, z_{q} \in \mathbb{C} \backslash \mathbb{R}$ are also pairwise different.

Assume that $P(x)$ is another polynomial such that $\operatorname{deg}(P)<\operatorname{deg}(Q)$. In this case, the real partial fraction decompositions of the rational function

$$
\frac{P(x)}{Q(x)}
$$

looks as follows:

$$
\frac{P(x)}{Q(x)}=\sum_{i=1}^{p} \sum_{r=1}^{k_{i}} \frac{a_{i r}}{\left(x-x_{i}\right)^{r}}+\sum_{j=1}^{q} \sum_{s=1}^{l_{j}} \frac{\beta_{j s} x+\gamma_{j s}}{\left.x^{2} 2 \operatorname{Re}\left(z_{j}\right) x+\left|z_{j}\right|^{2}\right)^{s}}
$$

where $a_{i r}, \beta_{j s}, \gamma_{j s} \in \mathbb{R}$.

One important problem of these PFDs is to determine the corresponding coefficients. To determine the coefficients of these PFDs, some methods/algorithms can be applied with respect to the given rational functions. For example, long division (routine calculation), algorithms introduced in $[1,2]$ can be used for suitable applications. These algorithms especially focus for determining the real PFDs for given rational functions. On the other hand, real PFDs may fail in some applications; for example a failure of real PFD is mentioned in [3]. From the point of view, complex PFD can alternatively be used and this yields simplest form for representation of rational function (1). In this paper, we aim to expose the full complex PFDs of rational function
(1). We first give some complex partial fraction decompositions and then emphasize the relationship between the coefficients of real PFD and complex PFD. Then, some applications of these complex PFDs will be discussed.

\section{Complex Partial Fraction Decompositions}

Complex PFDs can effectively be used in the parts of suitable applications. For example, rational algorithm, which is introduced in [3] for computing the coefficients of formal power series of a rational functions, requires complex section we provide the relations between the coefficients of real PFD and the coefficients of their complex PFD. Note that to find corresponding real PFDs, the algorithms discussed in $[1,2]$ can be used for suitable applications.

\section{Theorem 1}

$$
\begin{aligned}
& \text { If } z \in \mathbb{C} \backslash \mathbb{R}, \omega=\frac{1}{2 i \operatorname{Im}(z)} \in \mathbb{C} \text { and } R_{k}(x)=\frac{\omega^{k}}{(x-z)^{k}}+\frac{\omega^{k}}{(x-z)^{k}} \text {, where } k \text { is a positive integer, then } \\
& \text { 1. } R_{k} R_{1}=R_{k+1}+\omega \bar{\omega} R_{k-1} R_{1}, \text { forall } k \geq 2 \text { and } R_{1}^{2}=R_{2}+2 \omega \bar{\omega} R_{1} \\
& \text { 2. } R_{j} R_{1}=R_{j+1}+\omega \bar{\omega} R_{j}+\omega^{2} \bar{\omega}^{2} R_{j-1}+\ldots \omega^{j-1} \bar{\omega}^{j-1} R_{2}+2 \omega^{j} \bar{\omega}^{j-1} R_{2}+2 \omega^{j} \bar{\omega}^{j} R_{1}, \text { for all } j \geq 2 \\
& \text { 3.the rational funcion } R_{1}^{q}(x)=\frac{1}{\left(x^{2}-2 \operatorname{Re}(z) x+|z|^{2}\right)^{q}} \text { can be represented as } \\
& R_{1}^{q}=R_{q}+C_{q}^{1} \omega \bar{\omega} R_{q-1}+C_{q+1}^{2} \omega^{2} \bar{\omega}^{2} R_{q-2}+\ldots+C_{2 q-2}^{q-1} \omega^{q-1} \bar{\omega}^{q-1} R_{1}
\end{aligned}
$$

Proof 1: (1) we have successively:

$$
\begin{aligned}
R_{k} R_{1} & =\left(\frac{\omega^{k}}{(x-z)^{k}}+\frac{\bar{\omega}^{k}}{(x-\bar{z})^{k}}\right)\left(\frac{\omega}{(x-z)}+\frac{\bar{\omega}}{(x-\bar{z})}\right) \\
& =\frac{\omega^{k+1}}{(x-z)^{k+1}}+\frac{\omega^{k+1}}{(x-\bar{z})^{k+1}}+\frac{\omega^{k} \bar{\omega}}{(x-z)^{k}(x-\bar{z})}+\frac{\omega \bar{\omega}^{k}}{(x-z)(x-\bar{z})^{k}} \\
& =\frac{\omega^{k+1}}{(x-z)^{k+1}}+\frac{\bar{\omega}^{k+1}}{(x-\bar{z})^{k+1}}+\frac{\omega \bar{\omega}}{(x-z)(x-\bar{z})}\left(\frac{\omega^{k-1}}{(x-z)^{k-1}}+\frac{\omega^{k-1}}{(x-\bar{z})^{k-1}}\right) \\
& =R_{k+1}+\omega \bar{\omega} R_{k-1} R_{1}
\end{aligned}
$$

*Corresponding author: Ali Özyapici, Girne American University, Department of Engineering, Kyrenia, Mersin 10, Turkey, E-mail: ali.ozyapici@emu.edu.tr

Received September 22, 2012; Accepted November 24, 2012; Published November 27, 2012

Citation: Özyapici A, Pintea CS (2012) Complex Partial Fraction Decompositions of Rational Functions. J Applied Computat Mathemat 1:120. doi:10.4172/21689679.1000120

Copyright: @ 2012 Özyapici A, et al. This is an open-access article distributed under the terms of the Creative Commons Attribution License, which permits unrestricted use, distribution, and reproduction in any medium, provided the original author and source are credited. 
Moreover,

$$
\begin{aligned}
R_{1}^{2} & =\left(\frac{\omega}{x-z}+\frac{\bar{\omega}}{x-z}\right)^{2} \\
& =\frac{\omega^{2}}{(x-z)^{2}}+\frac{\bar{\omega}^{2}}{(x-\bar{z})^{2}}+\frac{2 \omega \bar{\omega}}{(x-z)(x-\bar{z})} \\
& =\frac{\omega^{2}}{(x-z)^{2}}+\frac{\bar{\omega}^{2}}{(x-\bar{z})^{2}}+2 \omega \bar{\omega}\left(\frac{\omega}{x-z}+\frac{\bar{\omega}}{x-z}\right) \\
& =R_{2}+2 \omega \bar{\omega} R_{1}
\end{aligned}
$$

(2) We first observe that

$$
R_{k} R_{1}=R_{k+1}+a R_{k-1} R_{1} \Leftrightarrow \frac{R_{k} R_{1}}{a^{k}}-\frac{R_{k-1}}{a^{k}} \text {, where } a=\omega \bar{\omega}=|\omega|^{2}
$$

By assigning successively the values $2 ; 3 ;::: ; j$ to $\mathrm{k}$, one gets:

$$
\begin{aligned}
& \frac{R_{2} R_{1}}{\omega^{2} \bar{\omega}^{2}}-\frac{R_{1} R_{1}}{a}=\frac{R_{3}}{a^{2}} \\
& \frac{R_{3} R_{1}}{a^{3}}-\frac{R_{3} R_{1}}{a^{2}}=\frac{R_{4}}{a^{3}} \\
& \vdots \\
& \frac{R_{j} R_{1}}{a j}-\frac{R_{j-1} R_{1}}{a^{j-1}}=\frac{R_{j+1}}{a^{j}},
\end{aligned}
$$

which shows that

$\frac{R_{j} R_{1}}{a^{j}}-\frac{R_{1} R_{1}}{a}=\frac{R_{3}}{a^{2}}+\frac{R_{4}}{a^{3}}+\ldots+\frac{R_{j+1}}{a^{J}}$

that is

$R_{j} R_{1}=a^{j-1} R_{1}^{2}+a^{j-2} R_{3}+\ldots+a R_{j}+a R_{j}+R_{j+1}$.

Since $R_{1}^{2}=R_{2}+2 a R_{1}$, the announced relation

follows now easily.

(3) We first observe that

$$
\frac{1}{x^{2}-2 \operatorname{Re}\left(z_{j}\right) x+|z|^{2}}=\frac{\omega}{x-z}+\frac{\bar{\omega}}{x-\bar{z}}=R_{1} \text {, }
$$

which implies that

$$
\frac{1}{\left(x^{2}-2 \operatorname{Re}\left(z_{j}\right) x+|z|^{2}\right)^{q}}=\left(\frac{\omega}{x-z}+\frac{\bar{\omega}}{x-z}\right)^{q}=R_{1}^{q} \text {. }
$$

The proof is done by induction on $\mathrm{q}$. If $\mathrm{q}=1$ or $\mathrm{q}=2$ one gets the obvious equalities

$$
R_{1}=R_{2} \text { and } R_{1}^{2}=R_{2}+2 a R_{1} \text {. }
$$

Assume the equality for $\mathrm{q}-1$ and observe that one gets successively:

$$
\begin{aligned}
R_{1}^{q}= & R_{1}^{q-1} R_{1} \\
= & \left(R_{q-1}+a C_{q-1}^{1} R_{q-2}+a^{2} C_{q}^{2} R_{q-3}+\ldots+a^{q-2} C_{2 q-4}^{q-2} R_{1}\right) R_{1} \\
= & R_{q-1} R_{1}+a C_{q-1}^{1} R_{q-2} R_{1}+a^{2} C_{q}^{2} R_{q-3} R_{1}+\ldots+a^{q-2} C_{2 q-4}^{q-2} R_{1} R_{1} \\
= & R_{q}+a R_{q-1}+a^{2} C_{q-1}^{1} R_{q-2}+\ldots+a^{q-2} C_{q-1}^{1} R_{2}+2 a^{q-1} \\
& C_{q-1}^{1} R_{1}+a^{2} C_{q}^{2} R_{q-2 q}+\ldots+a^{q-2} C_{q}^{2} R_{2}+2 a^{q-1} C_{q}^{2} R_{1} \\
& a^{q-2} C_{2 q-4}^{q-2} R_{2}+2 a^{q-1} C_{2 q-4}^{q-2} R_{1}
\end{aligned}
$$

$$
\begin{aligned}
& =R_{q}+a\left(C_{q-1}^{0}+C_{q-1}^{1}\right) R_{q-1}+a^{2}\left(C_{q-1}^{0}+C_{q-1}^{0}+C_{q}^{2}\right) R_{q-2}+\ldots+ \\
& +a^{q-2}\left(C_{q-1}^{0}+C_{q-1}^{1}+\ldots+C_{2 q-4}^{q-2}\right) R_{2}+2 a^{q-1}\left(C_{q-1}^{0}+C_{q-1}^{1}+\ldots+C_{2 q-4}^{q-2}\right) R_{1} .
\end{aligned}
$$

By using the formula $C_{n}^{k}=C_{n-1}^{k}+C_{n-1}^{k-1}$, one gets

$$
\begin{aligned}
& \begin{aligned}
& C_{q-1}^{0}+C_{q-1}^{1}=C_{q}^{1} \\
& C_{q-1}^{0}+C_{q-1}^{1}+C_{q}^{2}=C_{q}^{1}+C_{q}^{2}=C_{q+1}^{2} \\
& C_{q-1}^{0}+C_{q-1}^{1}+C_{q}^{2}+\ldots+C_{2 q-4}^{q-2}=C_{q}^{1}+C_{q}^{2}+\ldots+C_{2 q-4}^{q-2} \\
&=C_{q+1}^{2}+\ldots+C_{2 q-4}^{q-2} \\
&=C_{2 q-3}^{q-2}
\end{aligned} \\
& \text { Consequently }
\end{aligned}
$$

$$
\begin{aligned}
& R_{1}^{q}=R_{q}+a\left(C_{q-1}^{0}+C_{q-1}^{1}\right) R_{q-1}+a^{2}\left(C_{q-1}^{0}+C_{q-1}^{1}+C_{q}^{2}\right) R_{q-2}+\ldots+a^{q-2} \\
& \left(C_{q-1}^{0}+C_{q-1}^{1}+\ldots+C_{2 q-4}^{q-2}\right) R_{2}+2 a^{q-1}\left(C_{q-1}^{0}+C_{q-1}^{1}+\ldots+C_{2 q-4}^{q-2}\right) R_{1}= \\
& \quad=R_{q}+a C_{q}^{1} R_{q-1}+a^{2} C_{q+1}^{2} R_{q-2}+\ldots+a^{q-2} C_{2 q-3}^{q-2} R_{2}+2 a^{q-1} C_{2 q-3}^{q-2} R_{1}= \\
& R_{q}+a C_{q}^{1} R_{q-1}+a^{2} C_{q+1}^{2} R_{q-2}+\ldots+a^{q-2} C_{2 q-3}^{q-2} R_{2}+a^{q-1}\left(C_{2 q-3}^{q-2}+C_{2 q-3}^{q-1}\right) R_{1}= \\
& =R_{q}+a C_{q}^{1} R_{q-1}+a^{2} C_{q+1}^{2} R_{q-2}+\ldots+a^{q-2} C_{2 q-3}^{q-2} R_{2}+a^{q-1} C_{2 q-2}^{q-1} R_{1}
\end{aligned}
$$

Now the new algorithms for finding the desired complex coefficients of a certain type of rational function with respect to corresponding PFDs are given by the following proposition and corollary:

\section{Proposition 1}

$$
\begin{aligned}
& z \in \mathbb{C} \backslash \mathbb{R} \text { and } \beta_{1}, \gamma_{1}, \ldots, \beta_{1}, \gamma_{l} \in \text {, then } \\
& \sum_{s=1}^{l} \frac{\beta_{s} x+\gamma_{s}}{\left(x^{2}-1 \operatorname{Re}(z) x+|z|^{2}\right)^{s}}=\sum_{s=1}^{l}\left(\frac{b_{s}}{(x-z)^{s}}+\frac{\bar{b}_{s}}{(x-\bar{z})^{s}}\right),
\end{aligned}
$$

where

$$
\begin{aligned}
b_{1} & =\sum_{s=1}^{l} \beta_{s} \omega^{2}|\omega|^{2(s-2)} C_{2 s-4}^{s-2}+\sum_{s=2}^{l}\left(\beta_{s} \omega z+\omega \gamma_{s}\right)|\omega|^{2(s-1)} C_{2(s-1),}^{s-1} \\
b_{2} & =\sum_{s=2}^{l} \beta_{s} \omega^{3}|\omega|^{2(s-3)} C_{2 s-4}^{s-3}+\sum_{s=2}^{l}\left(\beta_{s} \omega^{2} z+\omega^{2} \gamma_{s}\right)|\omega|^{2(s-2)} C_{2 s-3}^{s-2}, \\
& \vdots \\
b_{l-1} & =\beta_{l} \omega^{l}+\beta_{l-1} \omega^{l-1} z+\omega^{l-1} \gamma_{l-1}+\left(\beta_{l} \omega^{l-1} z+\omega^{l-1} \gamma_{l}\right) \mid \omega^{2} C_{l}^{1} \\
b_{l} & =\beta_{l} \omega^{l} z+\omega^{l} \gamma_{l} .
\end{aligned}
$$

Proof 2: Considering for the rational function $S=\sum_{s=1}^{l} \frac{\beta_{s} x+\gamma_{s}}{\left(x^{2}-2 \operatorname{Re}(z) x+|z|^{2}\right)^{s}}$ one gets successively:

$$
\begin{aligned}
S & =\sum_{s=1}^{l}\left(\beta_{s} x+\gamma_{s}\right) R_{1}^{s} \\
& =\sum_{s=1}^{l}\left(\beta_{s} x+\gamma_{s}\right) \sum_{i=0}^{s-1} C_{s+i-1}^{i}|w|^{2 i} R_{s-i} \\
& =\sum_{s=1}^{l} \sum_{i=0}^{s-1}\left(\beta_{s} x+\gamma_{s}\right)|\omega|^{2 i} C_{s+i-1}^{i}\left(\frac{\omega^{s-1}}{(x-z)^{s-i}}+\frac{\bar{\omega}^{s-i}}{(x-\bar{z})^{s-i}}\right) \\
& =\sum_{s=1}^{l} \sum_{i=0}^{s-2}\left(\frac{\beta_{s} \omega^{s-i}|\omega|^{2 i} C_{s+i-1}^{i}}{(x-z)^{s-i-1}}+\frac{\beta_{s} \bar{\omega}^{s-i}|\omega|^{2 i} C_{s+i-1}^{i}}{(x-\bar{z})^{s-i-1}}\right) \\
& +\sum_{s=1}^{l} \sum_{i=0}^{s-1}\left(\frac{\left.\left(\beta \omega^{s-i} z+\omega^{s-i} \gamma_{s}\right)|\omega|^{2 i} C_{s+i-1}^{i}+\frac{\left(\beta_{s} \bar{\omega}^{s-i} \bar{z}+\bar{\omega}^{s-i} \gamma_{s}\right)|\omega|^{2 i} C_{s+i-1}^{i}}{(x-z)^{s-i}}\right)}{(x-\bar{z})^{s-i}}\right. \\
& =\frac{b_{1}}{x-z}+\frac{b_{1}}{x-\bar{z}}+\frac{b_{2}}{(x-z)^{2}}+\frac{\bar{b}_{2}}{(x-\bar{z})^{2}}+\ldots+\frac{b_{l}}{(x-z)^{l}}+\frac{\bar{b}_{l}}{(x-\bar{z})^{l}}
\end{aligned}
$$




$$
\begin{aligned}
& \text { Hence } \\
& b_{1}=\sum_{s=2}^{l} \beta_{s} \omega^{2}|\omega|^{2(s-2)} C_{2 s-3}^{s-2}+\sum_{s=1}^{l}\left(\beta_{s} \omega z+\omega \gamma_{s}\right)|\omega|^{2(s-1)} C_{2(s-1)^{\prime}}^{s-1} \\
& b_{2}=\sum_{s=3}^{l} \beta_{s} \omega^{3}|\omega|^{2(s-3)} C_{2 s-4}^{s-3}+\sum_{s=2}^{l}\left(\beta_{s} \omega^{2} z+\omega^{2} \gamma_{s}\right)|\omega|^{2(s-2)} C_{2 s-3}^{s-2} \\
& b_{l-1}=\beta_{l} \omega^{l}+\beta_{l-1} \omega^{l-1} z+\omega^{l-1} \gamma_{l-1}+\left(\beta_{l} \omega^{l-1} z+\omega^{l-1} \gamma_{l}\right)|\omega|^{2} C_{j}^{1} \\
& b_{l}=\beta_{l} \omega^{l} z+\omega^{l} \gamma_{l} .
\end{aligned}
$$

\section{Corollary}

Let $x_{1}, \ldots ., x_{p}$ be pairwise different real numbers and $z_{1}, \ldots ., z_{q} \in \mathbb{C} \backslash \mathbb{R}$ be also pairwise different. If is a polinomial with real coefficients whose degree satisfies the inequality $\operatorname{deg}(P(x))<p+2\left(l_{l}+\ldots+l_{q}\right)$, then there exists $a_{i r}, \beta_{j s}, \gamma_{j s} \in \mathbb{R}$ and $b_{j s} \in \mathbb{C}$ such that

$$
\frac{P(x)}{Q(x)}=\sum_{i=1}^{p} \sum_{r=1}^{k_{i}} \frac{a_{i r}}{\left(x-x_{i}\right)^{r}}+\sum_{j=1}^{q} \sum_{s=1}^{l_{j}}\left(\frac{b_{j s}}{\left(x-z_{j}\right)}+\frac{\bar{b}_{j s}}{\left(x-\bar{z}_{j}\right)^{s}}\right),
$$

\section{where}

$Q(x)=\left(x-x_{1}\right)^{k_{1}} \ldots\left(x-x_{p}\right)^{k_{p}}\left(x^{2}-2 \operatorname{Re}\left(z_{1}\right) x+\left|z_{1}\right|^{2}\right)^{l_{1}} \ldots\left(x^{2}-2 \operatorname{Re}\left(z_{q}\right) x+\left|z_{q}\right|^{l_{q}}\right.$.

The relations between the coefficients of the real partial fraction decomposition and the coefficients of the complex partial fraction decomposition are:

$$
\begin{aligned}
& b_{j 1}=\sum_{s=2}^{l} \beta_{j s} \omega_{j}^{2}|\omega|^{2(s-2)} C_{2 s-3}^{s-2}+\sum_{s=1}^{l}\left(\beta_{j s} \omega_{j} z+\omega_{j} \gamma_{j s}\right)\left|\omega_{j}\right|^{2(s-1)} C_{2(s-1)^{\prime}}^{s-1} \\
& b_{j 2}=\sum_{s=3}^{l} \beta_{j s} \omega_{j}^{3}\left|\omega_{j}\right|^{2(s-3)} C_{2 s-4}^{s-3}+\sum_{s=2}^{l}\left(\beta_{j s} \omega^{2} z+\omega_{j}^{2} \gamma_{j s}\right)\left|\omega_{j}\right|^{2(s-2)} C_{2 s-3^{\prime}}^{s-2} \\
& b_{j l_{j}-1}=\beta_{j l_{j}} \omega^{l}+\beta_{j l_{j}-1} \omega_{j}^{l_{j}-1} z+\omega_{j}^{l_{j}-1} \gamma_{j} l_{j}-1+\left(\beta_{j l_{j}} \omega_{j}^{l_{j}-1} z+\omega_{j}^{l_{j}-1} \gamma_{j l_{j}}\right)\left|\omega_{j}\right|^{2} C_{l_{j}}^{1} \\
& b_{j l j}=\beta_{j l_{j}} \omega_{j}^{l} z+\omega_{j}^{l} \gamma_{j l_{j}}, \\
& \text { where } \omega_{j}=\frac{1}{2 i \operatorname{Im}\left(z_{j}\right)}
\end{aligned}
$$

\section{Example 1}

Consider the rational function

$$
f(x)=\frac{2 x+1}{\left(x^{2}+4 x+5\right)\left(x^{2}+x+2\right)} .
$$

For the first step we need to find corresponding PFD of given function. The given rational function is decomposed in [3] by "Two Brick Method" as

$$
f(x)=\frac{1}{6}\left(\frac{-x-7}{\left(x^{2}+4 x+5\right)}+\frac{x+4}{\left(x^{2}+x+2\right)}\right) .
$$

Then, according to the Proposition (1) the complex PFDs of decomposed functions are obtained respectively as,

$$
\begin{aligned}
& \frac{-x-7}{\left(x^{2}+4 x+5\right)}=\frac{\left(-\frac{1}{2}+\frac{5 j}{2}\right)}{x+(2-i)}-\frac{\left(\frac{1}{2}+\frac{5 i}{2}\right)}{x+(2+i)} \text { and } \\
& \frac{x+4}{\left(x^{2}+x+2\right)}=\frac{\left(-\frac{\sqrt{7}}{2}-\frac{7 i}{2}\right)}{x+\left(-\frac{1}{2}+\sqrt{\frac{7 i}{4}}\right)}+\frac{\left(-\frac{\sqrt{7}}{2}+\frac{7 i}{2}\right)}{x+\left(-\frac{1}{2}-\sqrt{\frac{7}{4}} i\right)} .
\end{aligned}
$$

Thus,

$$
\frac{2 x+1}{\left(s^{2}+4 x+5\right)\left(x^{2}+x+2\right.}=\frac{1}{6}\left(\frac{\left(-\frac{1}{2}+\frac{5 i}{2}\right.}{x+(2-i)}-\frac{\left(\frac{1}{2}+\frac{5 i}{2}\right.}{x+(2+i)}+\frac{\left(-\frac{\sqrt{7}}{2}-\frac{7 i}{2}\right)}{x+\left(-\frac{1}{2}+\sqrt{\frac{7}{4}} i\right)}+\frac{\left(-\frac{\sqrt{7}}{2}+\frac{7 i}{2}\right.}{x+\left(-\frac{1}{2}-\sqrt{\frac{7}{4}} i\right)}\right)
$$

\section{Example 2}

Apply complex PFD of a more complicated rational function

$$
f(x)=\frac{2 x+1}{\left(x^{2}+6 x+10\right)^{3}} .
$$

Using proposition 2, the desired complex PFDs with corresponding complex coefficients can be given as

$$
\frac{2 x+1}{\left(x^{2}+6 x+10\right)^{3}}=\sum_{s=1}^{3}\left(\frac{b_{s}}{(x-(-3+i))^{s}}+\frac{\bar{b}_{s}}{(x-(-3-i))^{s}}\right)
$$

where $b_{1}=\frac{15 i}{16}, b_{2}=\frac{15}{16}-\frac{i}{8}$ and $b_{3}=\frac{-1}{4}-\frac{5 i}{8}$.

\section{Differentiation via Complex Partial Fraction Decompositions}

Higher order derivatives of functions can be used in many applications. Hovewer, representing the higher order derivatives of many functions explicitly cannot be easy and in general they are computed recursively. From the point of view, higher order derivatives of a certain class of rational functions can easily be computed through complex PFDs and the $\mathrm{k}^{\text {th }}$ order derivatives can be computed directly. In this section we will give the general form of higher order derivatives of rational function (1). Finally, the results then will be used in some certain applications.

In order to find the high order derivatives of rational functions in (1), we suppose to find the high order derivatives of rational functions of types

$$
\frac{a}{\left(x-x_{0}\right)^{r}} \text { and } \frac{a x+\beta}{\left(x^{2}-2 \operatorname{Re}(z) x+|z|^{2}\right)^{s}}
$$

where $a, x_{0}, \alpha, \beta \in \mathbb{R}$. While computing high order derivatives of the first type of rational functions is direct, computing the high order derivatives of the other type of rational functions needs some more computations which will be given in this section.

If $z \in \mathbb{C}$ is a fixed complex number, then the function

$$
\mathbb{R} \backslash\{z\} \rightarrow \mathbb{C}, x \mapsto \frac{1}{(x-z)^{k}},
$$

with $\mathrm{k}$ an integer, is arbitrarily many times differentiable and

$$
\frac{d^{n}}{d x^{n}}\left(\frac{1}{(x-z)^{k}}\right)=\frac{(-1)^{n} A_{n+k-1}^{n}}{(x-z)^{n+k}},
$$

where $A_{m}^{p}$ stands for $\frac{m !}{(m-p)}=m(m-1) \ldots .(m-p+1)$ and $0 !=1$ Indeed, it can be easily done by induction on $\mathrm{n}$. Consequently

$$
\begin{aligned}
\frac{d^{n}}{d x^{n}}\left(\frac{a x+b}{(x-z)^{k}}\right) & =\frac{d^{n}}{d x^{n}}\left(\frac{a}{(x-z)^{k-1}}+\frac{a z+b}{(x-z)^{k}}\right) \\
& =a \frac{(-1)^{n} A_{n+k-2}^{n}}{(x-z)^{n+k-1}}+(a z+b) \frac{(-1)^{n} A_{n+k-1}^{n}}{(x-z)^{n+k}} .
\end{aligned}
$$

In particular 
Citation: Özyapici A, Pintea CS (2012) Complex Partial Fraction Decompositions of Rational Functions. J Applied Computat Mathemat 1:120. doi:10.4172/2168-9679.1000120

Page 4 of 5

$$
\frac{d^{n}}{d x^{n}}\left(\frac{a x+b}{x-z}\right)=(-1)^{n} n ! \frac{a z+b}{(x-z)^{n+1}} \text { and } \frac{d^{n}}{d x^{n}}\left(\frac{1}{x-z}\right)=\frac{(-1)^{n} n !}{(x-z)^{n+1}} .
$$

Corollary 6: The $\mathrm{n}^{\text {th }}$ order derivatives of the function $\mathrm{R}$ and $\mathrm{R}$, respectively, are given by the following formulae.

$$
\begin{aligned}
& \text { 1. } R_{q}^{(n)}(x)=(-1)^{n} A_{n+q-1}^{n}\left(\frac{\omega^{q}}{(x-z)^{n+q}}+\frac{\bar{\omega}^{q}}{(x-\bar{z})^{n+q}}\right) \\
& =\frac{(-1)^{n} A_{n+q-1}^{n}}{2^{q-1}(\operatorname{Im}(z))^{q}} \frac{\sum_{k=0}^{n}(-1)^{\frac{k-q}{2}} C_{n+q}^{k}(x-\operatorname{Reven}(z))^{n+q-k}(\operatorname{Im}(z))^{k}}{\left(x^{2}-2 \operatorname{Re}(z) x+|z|^{2}\right)^{n+q}} \\
& \text { 2. } \frac{d^{n}}{d x^{n}}\left(\frac{1}{\left(x^{2}-2 \operatorname{Re}(z) x+|z|^{2}\right)^{q}}\right)=\sum_{k=0}^{q-1}|\omega|^{2 k} C_{q+k-1}^{k} \frac{d^{n}}{d x^{n}}\left(R_{q-k}(x)\right) \text {. }
\end{aligned}
$$

Proof: We just need to use the formula

$$
\frac{d^{n}}{d x^{n}}\left(\frac{1}{(x-z)^{q}}\right)=\frac{(-1)^{n} A_{n+q-1}^{n}}{(x-z)^{n+q}}
$$

and Theorem 1. Indeed, taking into account that $\omega=\frac{1}{2 \operatorname{Im}(z)}$, one gets successively:

$$
\begin{aligned}
& R_{q}^{(n)}(x)=(-1)^{n} A_{n+q-1}^{n}\left(\frac{\omega^{q}}{(x-z)^{n+q}}+\frac{\bar{\omega}^{q}}{(x-\bar{z})^{n+q}}\right) \\
& =(-1)^{n} A_{n+q-1}^{n}\left(\frac{\frac{1}{2^{q i q}(\operatorname{Im}(z))^{q}}}{(x-z)^{n+q}}+\frac{\frac{(-1)^{q}}{2^{q i q}(\operatorname{Im}(z))^{q}}}{(x-\bar{z})^{n+q}}\right) \\
& =\frac{(-1)^{n} A_{n+q-1}^{n}}{2^{q, q}(\operatorname{Im}(z))}\left(\frac{1}{\left(x-\operatorname{Re}(z)-i \operatorname{Im}(z)^{n+q}\right.}+\frac{(-1)^{q}}{(x-\operatorname{Re}(z)+i \operatorname{Im}(z))^{n+q}}\right) \\
& =\frac{(-1)^{n} A_{n+q-1}^{n}}{2^{q_{i q}}(\operatorname{Im}(z))^{q}} \frac{\sum_{k=0}^{n+q} C_{n+q}^{k}(x-\operatorname{Re}(z))^{n+q-k_{i} k}(\operatorname{Im}(z))^{k}\left[1+(-1)^{k+q}\right]}{\left(x^{2}-2 \operatorname{Re}(z) x+|z|^{2}\right)^{n+q}} \\
& =\frac{(-1)^{n} A_{n+q-1}^{n}}{2^{q-1}(\operatorname{Im}(z))^{q}} \frac{\sum_{\substack{k=0 \\
k+q-e v e n}}^{n+q} C_{n+q}^{k}(x-\operatorname{Re}(z))^{n+q-k_{k-q}}(\operatorname{Im}(z))^{k}}{\left(x^{2}-2 \operatorname{Re}(z) x+|z|^{2}\right)^{n+q}} \\
& =\frac{(-1)^{n} A_{n+q-1}^{n}}{2^{q-1}(\operatorname{Im}(z))^{q}} \frac{\sum_{\substack{k=0 \\
k+q-e v e n}}^{n+q} C_{n+q}^{k}(x-\operatorname{Re}(z))^{n+q-k_{i} k+q-2 q}(\operatorname{Im}(z))^{k}}{\left(x^{2}-2 \operatorname{Re}(z) x+|z|^{2}\right)^{n+q}} \\
& =\frac{(-1)^{n} A_{n+q-1}^{n}}{2^{q-1}(\operatorname{Im}(z))^{q}} \frac{\sum_{\substack{k=0 \\
k+q-e v e n}}^{n+q} C_{n+q}^{k}(x-\operatorname{Re}(z))^{n+q-k_{i} k+q-2 q}(\operatorname{Im}(z))^{k}}{\left(x^{2}-2 \operatorname{Re}(z) x+|z|^{2}\right)^{n+q}} \\
& \sum_{k=0}^{n+q} C_{n+q}^{k}(x-\operatorname{Re}(z))^{n+q-k}(-1) \frac{k+q}{2}(-1)^{-q}(\operatorname{Im}(z))^{k} \\
& =\frac{(-1)^{n} A_{n+q-1}^{n}}{2^{q-1}(\operatorname{Im}(z))^{q}} \frac{\sum_{\substack{k=0 \\
k+- \text {-even }}}}{\left(x^{2}-2 \operatorname{Re}(z) x+|z|^{2}\right)^{n+q}} \\
& =\frac{(-1)^{n} A_{n+q-1}^{n}}{2^{q-1}(\operatorname{Im}(z))^{q}} \frac{\sum_{\substack{k+0 \\
k+q-e v e n}}^{n+q}(-1)^{\frac{k-q}{2} C_{n+q}^{k}(x-\operatorname{Re}(z))^{n+q-k-k}(\operatorname{Im}(z))^{k}}}{\left(x^{2}-2 \operatorname{Re}(z) x+|z|^{2}\right)^{n+q}}
\end{aligned}
$$

Proposition 7: If $a$; $b$ are real numbers and $z$ is a complex number, then the following formula holds for every positive integer $n$

$$
\frac{d^{n} f}{d x^{n}}=n a \frac{d^{n-1}}{d x^{n-1}}\left(\frac{1}{\left(x^{2}-2 \operatorname{Re}(z) x+|z|^{2}\right)^{q}}\right)+(a x+b) \frac{d^{n}}{d x^{n}}\left(\frac{1}{\left(x^{2}-2 \operatorname{Re}(z) x+|z|^{2}\right)^{q}}\right)
$$

where $f$ is the rational function given by

$$
f(x)=\frac{a x+b}{\left(x^{2}-2 \operatorname{Re}(z) x+|z|^{2}\right) s^{q}}
$$

Proof: It follows immediately by induction on $\mathrm{n}$.

Corollary 8: If $R(x)=\frac{P(x)}{Q(x)}$ is the rational function as in corollary (3), then its nth order derivative is given by

$$
R^{(n)}(x)=\sum_{i=1}^{p} \sum_{r=1}^{k_{i}} \frac{(-1)^{n} a_{i r} A_{n+r-1}^{n}}{\left(x-x_{i}\right)^{n+r}}+\sum_{j=1}^{q} \sum_{s=1}^{l_{i}}\left(\frac{(-1)^{n} b_{j s} A_{n+s-1}^{n}}{\left(x-z_{j}\right)^{n+j}}+\frac{(-1)^{n} \bar{b}_{j s} A_{n+s-1}^{n}}{\left(x-\bar{z}_{j}\right)^{n+s}}\right)
$$

Consequently

$$
\left|R^{(n)}(x)\right| \leq \sum_{i=1}^{p} \sum_{r=1}^{k_{i}} \frac{\left|a_{i r}\right| A_{n+r-1}^{n}}{\left|x-x_{i}\right|^{n+r}}+2 \sum^{q} \sum_{j=1}^{l_{j}} \frac{\left|b_{j s}\right| A_{n+s-1}^{n}}{\left|x-z_{j}\right|^{n+s}} .
$$

\section{Some Applications Involve Higher Order Derivatives}

One of the important applications of complex PFDs is the differentiation of rational functions. The higher order derivatives of the rational function (1) can easily be computed by using the relation (2) and corollary 6 . In this section we discuss different problems involving higher order derivatives in which complex PFDs can be applied.

\section{Power series expansion}

According to the Taylor theorem, a function $f$ can be approximated by power series, if exists, about $\mathrm{x}_{0}$ as

$$
f(x)=\sum_{n=0}^{\infty} \frac{f^{(n)}\left(x_{0}\right)}{n !}\left(x-x_{0}\right)^{n}
$$

When $\mathrm{x}_{0}=0$ (Maclaurin series of $\mathrm{f}$ ), this series has the form

$$
f(x)=\sum_{n=0}^{\infty} \frac{f^{(n)}(0)}{n !}(x)^{n}=f(0)+\frac{f(0)}{1 !} x+\frac{f^{\prime \prime}(0)}{2 !} x^{2}+\ldots
$$

Thus the coefficients of Maclaurin series are represented by higher order derivatives:

$$
a_{k}=\frac{f^{(k)}(0)}{k !} \text { or } a_{k}=\frac{1}{k !} \lim _{x \rightarrow 0} f^{(k)}(x)
$$

Practically, it may be difficult to represent $a_{k}$ explicitly for all $\mathrm{k}$ for a certain class of functions. From the point of view, if the desired power series deals with a rational function, the complex PFDs can be applied to represent the coefficients explicitly by the following theorem:

Theorem 1: Let $f$ be a rational function of (1). The coefficients of the Maclaurin series of $f$ are

$$
\begin{aligned}
& a_{k}=\frac{1}{k !}\left\{\sum_{i=1}^{p} \sum_{r=1}^{k_{i}} \frac{(-1)^{k} a_{i r} A_{k+r-1}^{k}}{\left(-x_{i}\right)^{k+r}}+\sum_{j=1}^{q} \sum_{s=1}^{l_{j}}\left(\sum_{i=1}^{q-1} a_{k}|\omega|^{2 i} C_{q+i-1}^{i}\right)\right\}, \\
& \text { where } a_{k}=k \beta_{j s}\left(R_{q-i}^{(k-1)}(0)\right)+\gamma_{j s}\left(R_{q-i}^{(k)}(0) .\right.
\end{aligned}
$$


Proof: It is easily obtain from (1) and Proposition 7.

Example 1: Consider the rational function

$$
f(x)=\frac{1}{\left(x^{2}+2 x+2\right)^{3}}
$$

Then the complex number $\mathrm{z}=-1+\mathrm{i}$ and the coefficients of Maclaurin series of given function according to the Theorem 9 can be given as

$$
a_{k}=\frac{1}{k !}\left(\sum _ { i = 0 } ^ { 2 } | \omega | ^ { 2 i } C _ { 2 + i } ^ { i } \left[\frac{d^{k}}{d x^{k}}\left(R_{3-i}(0)\right)\right.\right.
$$

Thus, Maclaurin series of given rational function $f$ is

$$
f(x)=\sum_{k=0}^{\infty} a_{k} x^{k}=\frac{1}{8}-\frac{3}{8} x^{1}+\frac{9}{16} x^{2}-\frac{1}{2} x^{3}+\frac{3}{16} x^{4}+\frac{3}{16} x^{5}-\frac{13}{32} x^{6}+O\left(x^{7}\right)
$$

On the other hand, in which cases $a_{k}=0$ for all $\mathrm{k}$; the coefficients should alternatively be computed. This problem is processed by Computer Algebra Systems (CASs). In [4] new algorithms are introduced for finding hypergeometric power series of given function. As we mentioned before, rational algorithm is one of important algorithm for finding power series of rational functions in which complex PFD is the first step of the algorithm. Thus, our algorithm given in corollary (3) can be used for the first step of rational algorithm.

\section{Higher order schwarzian derivatives}

Schwarzian derivative of a function $f$ (real or complex valued) is defined by

$$
S(f)(x)=\frac{f^{\prime \prime \prime}}{f^{\prime}}-\frac{3}{2}\left(\frac{f^{\prime \prime \prime}}{f^{\prime}}\right)^{2}
$$

and it is invariant under linear fractional transformations. Schwarzian derivatives appears in different fields of mathematics such as complex analysis, differential equations, hypergeometric series as well as dynamical systems. Since Schwarzian derivative involve higher order ordinary derivatives and their combinations, the Schwarzian derivative as well as higher order Schwarzian derivatives for a certain class of functions cannot easily be derived in a closed form. Although the simplest class of functions for ordinary differentiation is polynomials, the closed form of Schwarzian derivatives of a polynomial function

$$
P_{n}(x)=a_{1} x^{n+2}+a_{2} x^{n+1}+\ldots+a_{n+3}
$$

is complicated comparing with ordinary one and it was given in [3] as

$$
\begin{aligned}
S\left(P_{n}\right)(x)= & \frac{(n+2) !(n+1) a_{1}\left(\frac{(n+2)}{2 !} a_{1} x^{2}+(n+1) ! a_{2} x+n ! a_{3}\right)}{\left.(n+1)\left(\frac{(n+2) !}{2 !} a_{1} x^{2}+(n+1) ! a_{2} x+n ! a_{3}\right)^{2}\right)} \\
& -\frac{(n+2)\left((n+2) ! a_{1} x+(n+1) ! a_{2}\right)^{2}}{(n+1)\left(\frac{(n+1) !}{2 !} a_{1} x^{2}+(n+1) ! a_{2} x+n ! a_{3}\right)^{2}}
\end{aligned}
$$

Since the formula (5) is a rational function of $\mathrm{x}$, it can directly be decomposed by complex PFDs algorithm for suitable part of applications whenever

$$
\frac{a_{3}}{a_{1}}>\frac{(n+1) a_{2}^{2}}{(n+2) a_{1}^{2}}
$$

Moreover the higher order Schwarzian derivatives for a function $\mathrm{f}$ were defined inductively in [6] as

$$
S_{n+1}(f)=\left(S_{n}(f)\right)^{\prime}-(n-1) S_{n}(f) \cdot \frac{f^{\prime \prime}}{f}, n \succ 4
$$

From the point of view, the higher order Schwarzian derivatives involve more complicated combinations of higher order ordinary derivatives. For example, second and third order Schwarzian derivatives of the function $\mathrm{f}$; according to the (6), are given [2] as

$$
\begin{aligned}
& s_{4}=\frac{f^{\prime \prime \prime}}{f^{\prime}}-6 \frac{f^{\prime \prime \prime} f^{\prime \prime}}{f^{\prime 2}}+6\left(\frac{f^{\prime \prime}}{f^{\prime}}\right)^{3}, \\
& s_{5}=\frac{f^{\prime \prime \prime \prime}}{f^{\prime \prime}}-10 \frac{f^{\prime \prime \prime \prime} f^{\prime \prime}}{f^{\prime} 2}-6\left(\frac{f^{\prime \prime \prime}}{f^{\prime}}\right)^{2}+48 \frac{f^{\prime \prime \prime} f^{\prime \prime 2}}{f^{\prime 3}} 36\left(\frac{f^{\prime \prime}}{f^{\prime}}\right)^{4}
\end{aligned}
$$

If the function $f$ is deal with the rational function (1); the complex PFD algorithms can be used to defined its Schwarzian derivative as well as higher order Schwarzian derivatives in a closed form. Let us consider a particular type of rational functions

$$
R(x)=\frac{\beta x+\alpha}{\left(x^{2}-2 \operatorname{Re}(z)+|z|^{2}\right)^{q}}
$$

Then the higher order derivatives of the rational function $R(x)$ according to complex PFDs is

$$
\begin{aligned}
f(n, q) & =\frac{d^{n}}{d x^{n}}(R)(x)=\frac{d^{n}}{d x^{n}}\left(\sum_{s=1}^{q}\left(\left(\frac{b_{s}}{(x-z)^{s}}+\frac{\bar{b}_{s}}{(x-\bar{z})^{s}}\right)\right)\right) \\
& =\sum_{s=1}^{q}\left[\frac{(-1)^{n} b_{s} A_{n+s-1}^{n}}{(x-z)^{s+n}}+\frac{(-1)^{n} \bar{b}_{s} A_{n+s-1}^{n}}{(x-\bar{z})^{s+n}}\right]
\end{aligned}
$$

Thus Schwarzian derivative of the rational function $\mathrm{R}(\mathrm{x})$ can be easily represented in the closed form as

$$
\begin{aligned}
S_{1}(f)(x)= & \frac{f(3, q)}{f(1, q)}-\frac{3}{2}\left(\frac{f(2, q)}{f(1, q)}\right)^{2}=\frac{\sum_{s=1}^{q}\left[\frac{(-1)^{n} b_{s}(s+2) !}{(x-z)^{s+3}}+\frac{(-1)^{n} b_{s}(s+2 !)}{(x-\bar{z})^{s+3}}\right]}{\sum_{s=1}^{q}\left[\frac{(-1)^{n} b_{s} s !}{(x-z)^{s+1}}+\frac{(-1)^{n} \bar{b}_{s} s !}{(x-\bar{z})^{s+1}}\right]} \\
& -\frac{3}{2}\left(\frac{\sum_{s=1}^{q}\left[\frac{(-1)^{n} b_{s}(s+1) !}{(x-z)^{s+2}}+\frac{(-1)^{n} \bar{b}_{s}(s+1) !}{(x-\bar{z})^{s+2}}\right]}{\sum_{s=1}^{q}\left[\frac{(-1)^{n} b_{s} s !}{(x-z)^{s+1}}+\frac{(-1)^{n} b_{s} s !}{(x-\bar{z})^{s+1}}\right]}\right)^{2}
\end{aligned}
$$

\section{References}

1. Bronstein M, Salvy B (1993) Full Partial Decomposition of Rational Functions International Conference on Symbolic and Algebraic Compuattion, Kiev, Ukraine.

2. Dorff M, Szynal J Higher Order Schwarzian Derivatives for Convex Univalent Functions (under review).

3. Hacibekiroglu G, Caglar M, Polatlioglu Y (2009) The higher order Schwarzian derivative: Its applications for chaotic behavior and new invariant sufficient condition of chaos. Nonlinear Analysis: Real World Applications 10: 1270-1275.

4. Koepf W (1992) Power Series in Computer Algebra. J Symb Comput 13: 581-603

5. Kovacic JJ (1986) An Algorithm for Solving Second Order Linear Homogeneous Differential Equations. J Symb Comput 2: 3-43.

6. Schippers E (2000) Distortion Theorems for Higher Order Schwarzian Derivatives of Univalent Functions. P Am Math Soc 128: 3241-3249.

7. Witula W, Slota D (2008) Partial Fraction Decompositions of Some Rational Functions. Appl Math Comput 210: 328-336. 